06

\title{
Световые пули с бесселевым поперечным сечением в среде углеродных нанотрубок
}

\author{
(C) А.М. Белоненко, И.С. Двужилов , Ю.В. Двужилова, М.Б. Белоненко \\ Волгоградский государственный университет, \\ 400062 Волгоград, Россия \\ ฯ e-mail: dvuzhilov.lya@volsu.ru \\ Поступила в редакцию 15.08.2021 г. \\ В окончательной редакции 13.12.2021 г. \\ Принята к публикации 15.12.2021 г.
}

Рассмотрено распространение трехмерных предельно коротких оптических импульсов (световых пуль) с бесселевым поперечным сечением в среде углеродных нанотрубок, помещенных в оптический резонатор. В результате численных расчетов установлено, что такие импульсы распространяются устойчиво с сохранением энергии в ограниченной области пространства, в том числе на больших временах порядка 100 ps.

Ключевые слова: предельно короткие оптические импульсы, нелинейная среда, световые пули, углеродные нанотрубки.

DOI: $10.21883 / \mathrm{OS} .2022 .03 .52170 .2642-21$

\section{Введение}

В настоящее время для нелинейной оптики существенный интерес представляют бездифракционные оптические пучки (с поперечным сечением Бесселя, Эйри и Матье), которые представляют собой, импульсы, распространяющиеся без изменения формы и амплитуды. Дифракционное расплывание импульсов является фундаментальной особенностью свободно распространяющихся оптических лучей, которые легко наблюдаются в повседневной жизни. Как известно, дифракция оказывает значительное влияние на предел оптического разрешения в различных отраслях, в том числе в микроскопии, литографии и фотографии, а также на предел точности спектрального анализа [1]. Таким образом, бездифракционные пучки имеют ряд преимуществ перед другими лучами сопоставимого поперечного размера, например сдерживание уширения формы и масштаба импульса во время его распространения.

В настоящей работе будут рассмотрены пучки Бесселя. Уникальные свойства данных пучков характеризуются тем, что луч может формироваться в нелинейных средах, показатель преломления которых слабо меняется периодическим образом в зависимости от длины [2]. Пучки Бесселя можно генерировать вдоль спиральных [3-5], змее- или зигзагообразных [6] траекторий. Методики генерации бездифракционных пучков типа Бесселя, которые могут распространяться по произвольным траекториям, были предложены в [7] и впоследствии наблюдались в [8].

В качестве среды распространения импульсов были выбраны углеродные нанотрубки (УНТ), обладающие уникальными физическими свойствами в оптическом диапазоне вследствие непараболичности закона дисперсии электронов $[9,10]$. Простота их строения, уникаль- ность свойств [11-15] способствуют развитию исследований в области распространения оптических импульсов, разработке оптических приборов на их основе, но основное качество, которым обладают УНТ, - возможность их использования в качестве среды для образования световых пуль [16-19]. Углеродные нанотрубки вызывают большой интерес у множества исследователей. Они получили обширное применение в нелинейной оптике, благодаря чему играют одну из ключевых ролей в разработке различных приборов оптики, нанофотоники и т.д.

Задача о распространение импульсов различной формы в нелинейной среде не является тривиальной. Хрестоматийным примером является „теорема площадей в одномерной задаче о самоиндуцированной прозрачности. Так, импульсы, имеющие „площадь“ более $2 \pi$, устойчивы, а импульсы меньшей площади распадаются дисперсионным образом. Под площадью импульса понимается интеграл от частоты Раби по времени в пределах $t=-\infty$ (когда импульса еще не было) до некоторого достаточно большого времени $t$ (когда импульс уже прошел) [20]. Очевидно, что в случае импульсов Бесселя, которые распространяются устойчиво в линейной среде за счет интерференции дифракционных волн от боковых лепестков, их динамика в нелинейной среде представляет отдельную задачу. Углеродные нанотрубки были выбраны как объект, в котором нелинейный отклик среды можно посчитать аналитически с любой наперед заданной точностью.

В настоящей работе УНТ образуют неоднородную среду, которая помещена в оптический резонатор. В резонаторе применяется внутрирезонаторная лазерная спектроскопия, и при многократном отражении импульса от его стенок происходят потери энергии импульса по причине дифракции [21]. Дополнительно следует отме- 
тить, что возникают эффекты, связанные с отражением от стенок цилиндрического резонатора. Пучок проходит через массив УНТ много раз за счет многократного отражения от стенок цилиндрического резонатора. Важным достоинством таких импульсов (резонаторных солитонов) является то, что, имея оптическую обратную связь, они могут распространяться даже тогда, когда в отсутствие резонатора та же нелинейная среда не поддерживает каких-либо пространственных солитонов.

Ранее авторами уже были проведены исследования, посвященные распространению предельно коротких оптических импульсов, в том числе поперечного сечения Бесселя, в средах УНТ [22-25], однако в настоящей работе впервые будет произведено численное моделирование динамики импульсов на больших временах (до $140 \mathrm{ps}$ ), что играет важную роль для прикладных задач. Также отличительной особенностью работы является то, что неоднородная среда УНТ помещена в условия оптического резонатора.

Таким образом, задача о распространении бездифракционного импульса с бесселевым сечением в среде упорядоченных УНТ, помещенных в оптический резонатор, играет важную роль не только с теоретической, но и с прикладной точки зрения.

\section{Основные уравнения}

В настоящей работе будут рассмотрены следующие приближения: наведенный импульсом заряд вдоль оси углеродных нанотрубок, а также электрическое поле подложки не учитываются. Характерное расстояние между нанотрубками и их размер во много раз меньше, чем размер пространственной области, в которой локализован оптический пучок Бесселя. Это позволяет считать ток распространенным по объему и применить приближение сплошной среды.

Предполагаемая система смоделирована таким образом, что трехмерный предельно короткий оптический импульс распространяется в неоднородной среде УНТ типа zig-zag в оптическом резонаторе, электрическое поле которого направлено вдоль оси нанотрубок (рис. 1).

Гамильтониан системы электронов имеет вид

$$
H=\gamma \sum_{j \sigma} a_{j \sigma}^{*} a_{j \sigma}+\text { c.c. }
$$

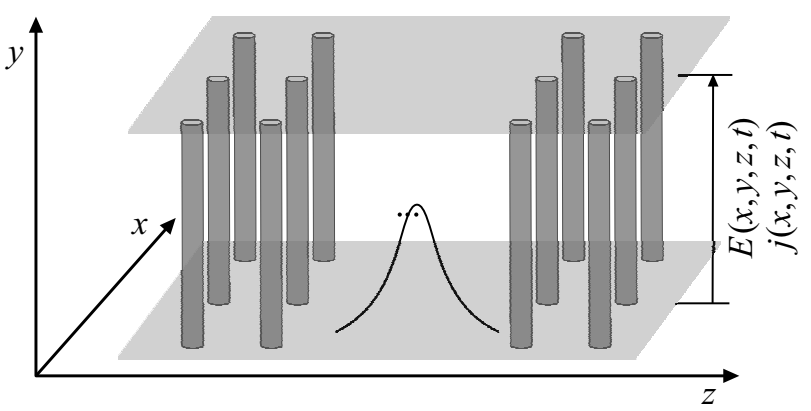

Рис. 1. Геометрия задачи. Ток и электрическое поле импульса направлены вдоль оси УНТ. где $a_{j \sigma}^{+}, a_{j \sigma}$ - операторы рождения и уничтожения электронов на узле $j$.

Запишем преобразование Фурье, которое диагонализирует гамильтониан $H$ :

$$
\begin{aligned}
& a_{n \sigma}^{+}=\frac{1}{\sqrt{N^{1 / 2}}} \sum_{j} a_{j \sigma}^{+} \exp (i j n), \\
& a_{n \sigma}=\frac{1}{\sqrt{N^{1 / 2}}} \sum_{j} a_{j \sigma} \exp (-i j n) .
\end{aligned}
$$

Спектр электронов, описывающий свойства электронной подсистемы в отсутствие кулоновского отталкивания УНТ типа „zig-zag“, имеет вид [26]

$$
\varepsilon_{s}(p)= \pm \gamma\left\{1+4 \cos (a p) \cos \left(\pi \frac{s}{m}\right)+4 \cos ^{2}\left(\pi \frac{s}{m}\right)\right\}^{1 / 2},
$$

где $s=1,2 \ldots m$, нанотрубка имеет тип $(m, 0)$, $\gamma \approx 2.7 \mathrm{eV}, a=3 b / 2 \hbar, b=0.142 \mathrm{~nm}$ - расстояние между соседними атомами углерода, $p$ - квазиимпульс.

Уравнения Максвелла, описывающие энергию предельно короткого оптического импульса с бесселевым поперечным сечением, можно записать в следующем виде [2,27]:

$$
\frac{\partial^{2} \mathbf{E}}{\partial x^{2}}+\frac{\partial^{2} \mathbf{E}}{\partial y^{2}}+\frac{\partial^{2} \mathbf{E}}{\partial z^{2}}-\frac{1}{c^{2}} \frac{\partial^{2} \mathbf{E}}{\partial t^{2}}+\frac{4 \pi}{c} \frac{\partial \mathbf{j}}{\partial t}+\frac{4 \pi}{c^{2}} \frac{\partial^{2} \mathbf{P}}{\partial t^{2}}=0,
$$

где $\mathbf{j}$ - плотность электрического тока, $c$ - скорость света в среде, $t$ - время, $\mathbf{E}-$ электрическое поле световой пули, $\mathbf{P}=\mu \mathbf{E}$, где $\mathbf{P}-$ вектор поляризации, параллельный вектору электрического поля импульса $\mathbf{E}$, $\mu$ - коэффициент линейной восприимчивости.

Модифицируем уравнение (4), учитывая кулоновскую калибровку для описания распространения импульсов с широким спектром в нелинейной среде:

$$
\mathbf{E}=-\frac{1}{c} \frac{\partial \mathbf{A}}{\partial t}
$$

Проинтегрировав уравнение (4) по времени, получим обобщенное уравнение на вектор-потенциал А в случае нелинейной среды:

$$
\frac{\partial^{2} \mathbf{A}}{\partial x^{2}}+\frac{\partial^{2} \mathbf{A}}{\partial y^{2}}+\frac{\partial^{2} \mathbf{A}}{\partial z^{2}}-\frac{1}{c^{2}}(1+4 \pi \mu) \frac{\partial^{2} \mathbf{A}}{\partial t^{2}}+\frac{4 \pi}{c} \mathbf{j}=0 .
$$

Вектор-потенциал А имеет вид $\mathbf{A}(0, A(r, z, t), 0)$, а плотность тока $-\mathbf{j}(0, j(r, z, t), 0)$. Запишем стандартное выражение для плотности тока в виде

$$
\mathbf{j}=e n(z, r) \sum_{p s} v_{s}\left(\mathbf{p}-\frac{e}{c} \mathbf{A}(t)\right)\left\langle a_{p s}^{+} a_{p s}\right\rangle
$$

где $v_{z}=\frac{\partial \varepsilon_{s}(\mathbf{p})}{\partial \mathbf{p}}$, скобки означают усреднение с неравновесной матрицей плотности $\rho(t):\langle B\rangle=S p(B(0) \rho(t))$. 

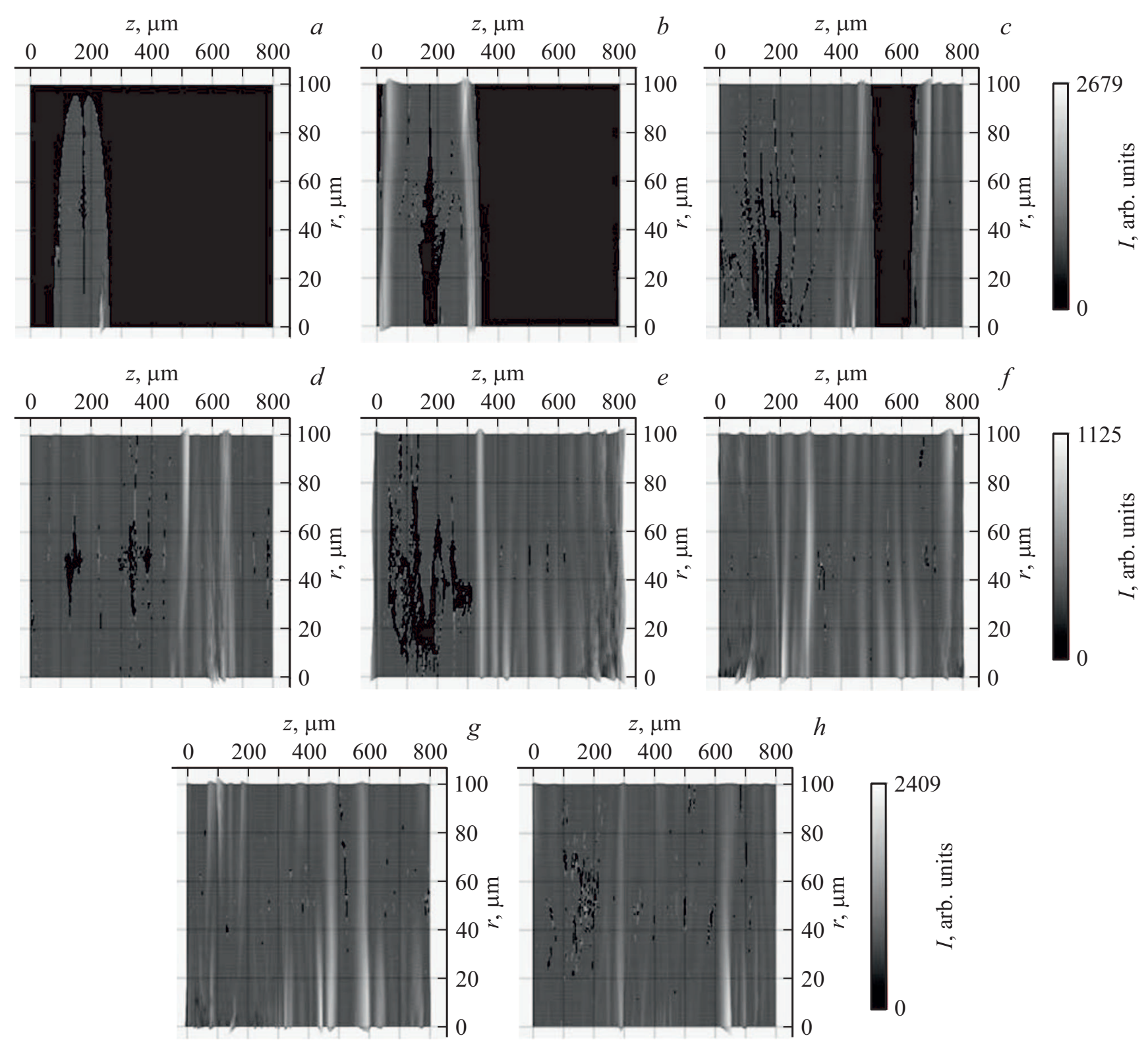

Рис. 2. Динамика световой пули с бесселевым поперечным сечением в неоднородной среде (период решетки $\chi=0.5 \mu \mathrm{m})$ УНТ, помещенных в оптический резонатор, в моменты времени $t=10(a), 20(b), 40(c), 60(d), 80(e), 100(f), 120(j), 140$ ps $(h)$.

Учитывая, что $\left[a_{p s}^{+} a_{p s}, H\right]=0$, из уравнений движения для матрицы плотности сразу получаем, что $\left\langle a_{p s}^{+} a_{p s}\right\rangle=\left\langle a_{p s}^{+} a_{p s}\right\rangle_{0}$, где $\langle B\rangle_{0}=S p(B(0) \rho(t)), n(z, r)-$ плотность УНТ [28].

С учетом того, что $\rho_{0}=\frac{\exp \left\{-H / k_{B} T\right\}}{S p(\langle B\rangle=S p(B(0) \rho(t)))}$, где $k_{B}-$ постоянная Больцмана, $T-$ температура, разложим $v_{s}(\mathbf{p})$ в ряд Фурье и получим

$$
\begin{aligned}
v_{s}\left(\mathbf{p}-\frac{e}{c} \mathbf{A}(t)\right)= & \sum_{k} A_{k s}\left(\sin (k \mathbf{p}) \cos \left(\frac{k e}{c} \mathbf{A}(t)\right)\right. \\
& \left.-\cos (k p) \sin \left(\frac{k e}{c} \mathbf{A}(t)\right)\right),
\end{aligned}
$$

где $A_{k s}=\int_{-\pi / a}^{\pi / a}=v_{s}(\mathbf{p}) \sin (k \mathbf{p}) d \mathbf{p}$ - убывающие с poстом $k$ коэффициенты разложения.

Если учесть то, что функция распределения $\rho_{0}-$ четная функция квазиимпульса $\mathbf{p}$, которая при усреднении $\mathrm{c} \sin (k \mathbf{p})$ даст ноль, уравнение (8) можно представить в виде

$$
v_{s}\left(\mathbf{p}-\frac{e}{c} \mathbf{A}(t)\right)=-\sum_{k} A_{k s} \cos (k \mathbf{p}) \sin \left(\frac{k e}{c} \mathbf{A}(t)\right) .
$$

Подставляя данное уравнение в (7) и производя суммирование по $p$ и $s$, получаем

$$
\mathbf{j}=-e n_{0} \sum_{k} B_{k} \sin \left(\frac{k e}{c} \mathbf{A}(t)\right)
$$



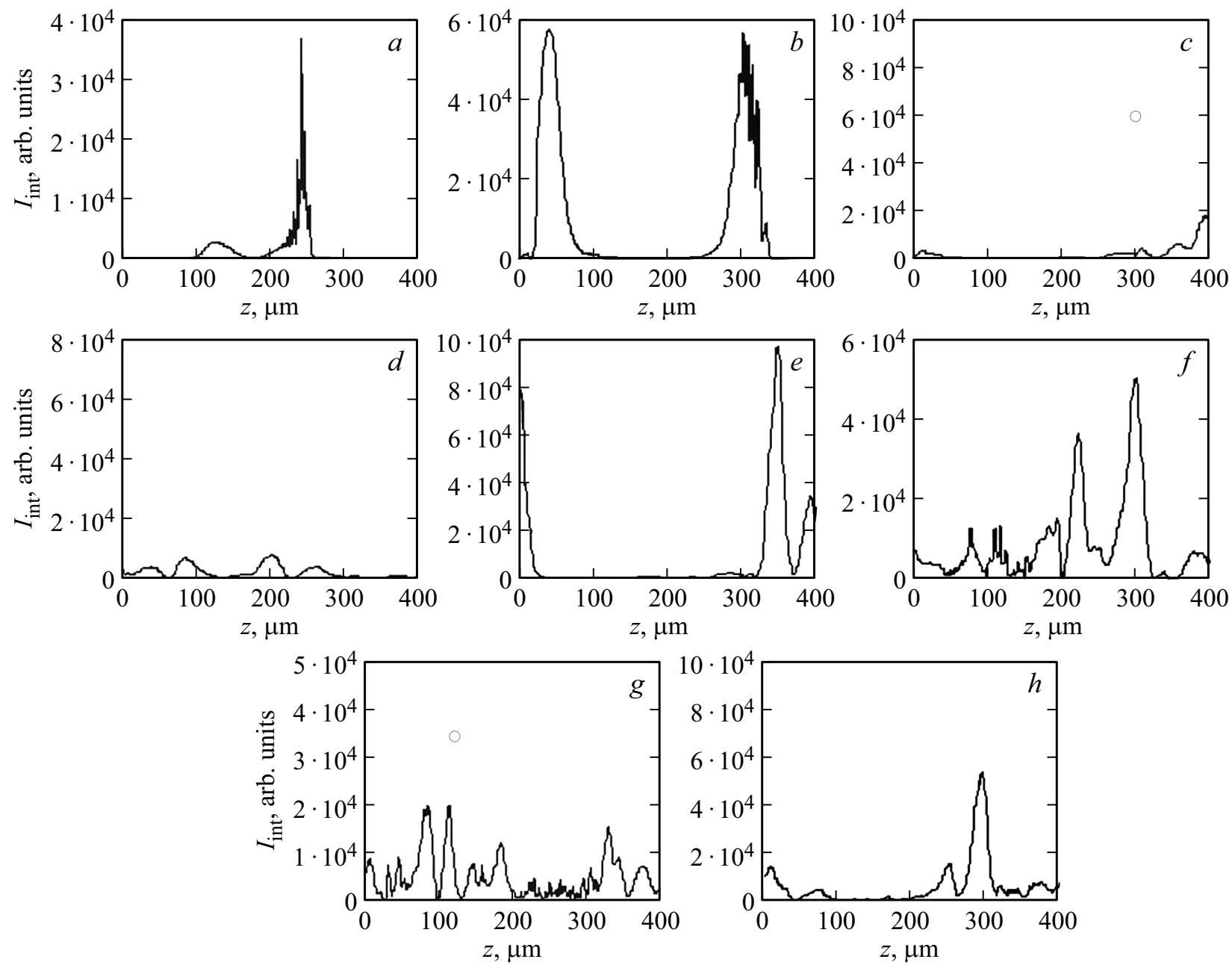

Рис. 3. Интегральная интенсивность по поперечному срезу резонатора в различные моменты времени $t=10(a), 20(b), 40(c)$, $60(d), 80(e), 100(f), 120(j), 140 \mathrm{ps}(h)$.

$$
B_{k}=\sum_{s=1-\pi_{-\pi / a}}^{m} \int_{k s}^{\pi / a} A_{k s} \cos (k \mathbf{p}) \frac{\exp \left\{-\beta \varepsilon_{s}(\mathbf{p})\right\}}{1+\exp \left\{-\beta \varepsilon_{s}(\mathbf{p})\right\}},
$$

где $n_{0}$ - концентрация равновесных электронов в УНТ, $\beta=1 / k_{B} T$.

Если учесть все вышесказанное, то уравнение (6) в цилиндрически симметричном случае после перехода к безразмерным величинам примет вид

$$
\frac{\partial^{2} \widetilde{\mathbf{A}}}{\partial z^{2}}+\frac{1}{r} \frac{\partial}{\partial r}\left(r \frac{\partial \widetilde{\mathbf{A}}}{\partial r}\right)-\frac{1}{c^{2}} \frac{\partial^{2} \widetilde{\mathbf{A}}}{\partial t^{2}}+\frac{4 \pi}{c} n(z, r) \widetilde{\mathbf{j}}=0
$$

где $r=\sqrt{x^{2}+y^{2}}$, слагаемое, зависящее от угла поворота, стремится к нулю, $\widetilde{\mathbf{A}}-$ безразмерный вектор-потенциал, $\widetilde{\mathbf{j}}-$ безразмерная плотность тока. Далее в наших расчетах коэффициент $n(z, r)$ будет задан в виде $n(z, r)=1+a \cos (2 \pi z / \chi)$, где $a$ задает глубину модуляции нелинейности, $\chi-$ период модуляции. В работе рассматривается модуляция вдоль оси $z$. Оптический резонатор представлен в цилиндрической форме и моделируется заданием периодических граничных условий вдоль оси цилиндра, а также введением граничных условий отражения на границе цилиндра. Отметим, что уравнение (11) является обобщением широко известного уравнения Синус-Гордона.

\section{Результаты исследования}

Начальные условия на вектор-потенциал электрического поля импульса задавались в следующем виде:

$$
\begin{aligned}
\mathbf{A}(z, r)= & Q \exp \left\{-\frac{\left(z-z_{0}\right)^{2}}{\gamma_{z}^{2}}\right\} J_{0}\left\{\left|\frac{\left(r-r_{0}\right)}{\gamma_{r}}\right|\right\} \\
& \times \exp \left\{-\Delta\left|r-r_{0}\right|\right\}, \\
\frac{d \mathbf{A}(z, r)}{d t}= & \frac{2 v z Q}{\gamma_{z}^{2}} \exp \left\{-\frac{\left(z-z_{0}\right)^{2}}{\gamma_{z}^{2}}\right\} J_{0}\left\{\left|\frac{\left(r-r_{0}\right)}{\gamma_{r}}\right|\right\} \\
& \times \exp \left\{-\Delta\left|r-r_{0}\right|\right\} .
\end{aligned}
$$

Здесь $Q$ - амплитуда импульса, $\Delta-$ параметр обрезания, который вводится в связи с тем, что пучок 

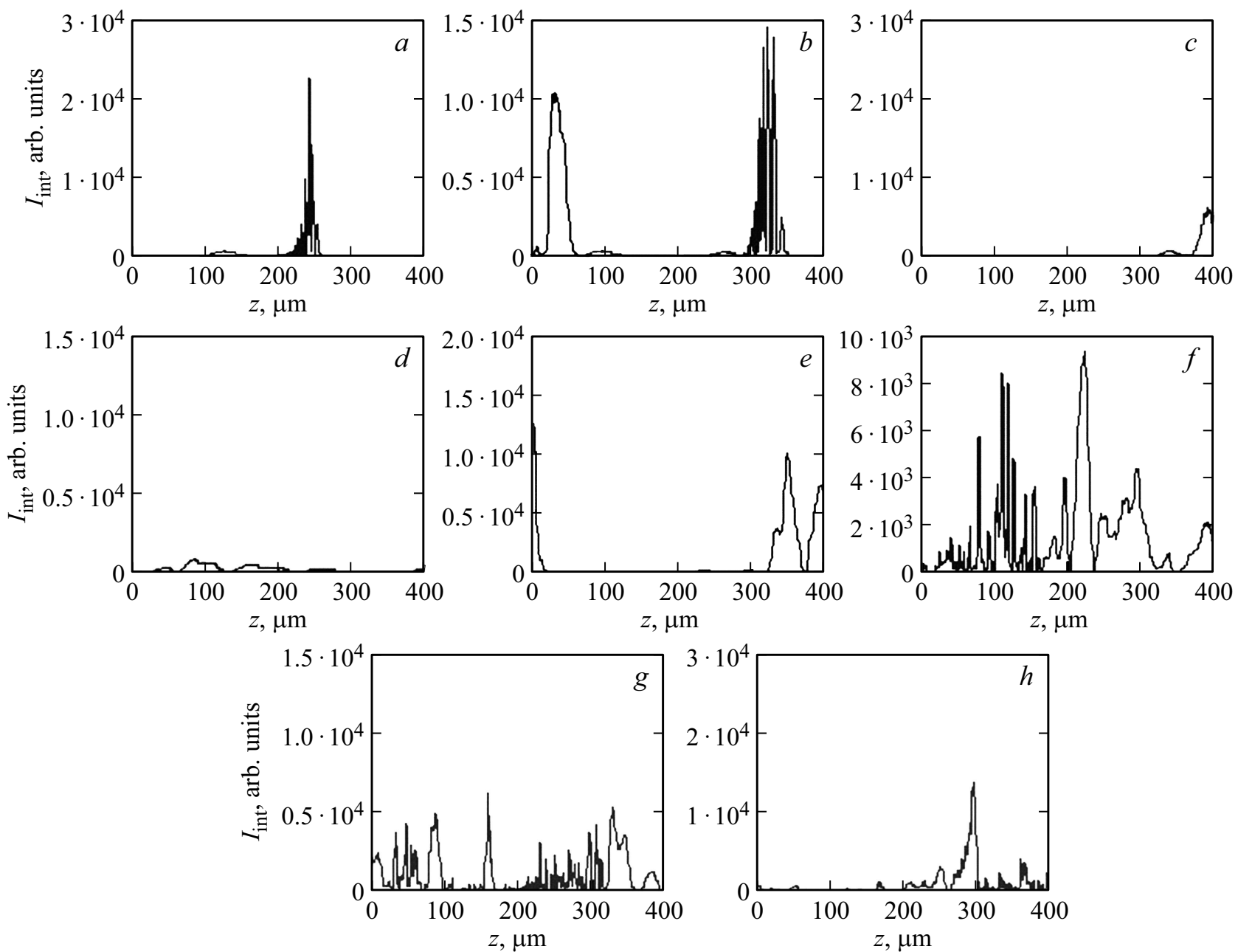

Рис. 4. Интегральная интенсивность вблизи оси цилиндрического резонатора в моменты времени $t=10(a), 20(b), 40(c), 60(d)$, $80(e), 100(f), 120(j), 140 \mathrm{ps}(h)$.

с бесселевым поперечным сечением физически нереализуем, и функцию необходимо обрезать на больших расстояниях для получения конечной энергии пучка, $\gamma_{z}, \gamma_{r}$ - ширина импульса в направлениях $z$ и $r, v-$ начальная скорость импульса.

Как показали результаты численных расчетов, представленные на рис. 2, распространение трехмерной световой пули с бесселевым поперечным сечением в среде упорядоченных УНТ, помещенных в оптический резонатор, является квазиустойчивым и квазистабильным, т.е. энергия электромагнитного импульса остается локализованной в ограниченной пространственной области.

Интенсивность электрического поля выражена в относительных единицах и пропорциональна квадрату электрического поля:

$$
\begin{gathered}
I(\text { arb. units }) \propto E^{2}=\left(E \frac{\omega_{0}}{c}\right)^{2}, \\
\omega_{0}=2|e| d_{x} h^{-1} \sqrt{\pi \gamma_{0} n} .
\end{gathered}
$$

Заметим, что все величины, входящие в выражение выше, измеряются в системе СИ.
Из представленных зависимостей видно, что световые пули с бесселевым поперечным сечением в неоднородной среде УНТ в условиях оптического резонатора распространяются квазиустойчиво, однако форма импульса все же подвержена дисперсионному расплыванию. Изменение формы наглядно продемонстрировано на рис. 3 и 4. Так, на рис. 3 показаны срезы интегралов по всему поперечному сечению резонатора от интенсивности, в то время как рис. 4 изображает интегралы по интенсивности по поперечному сечению вблизи оси интегрирования.

Значения соответствующего вектора Пойнтинга представлены на рис. 5, где проиллюстрировано уширение импульса вдоль радиального направления (по направлению к стенкам резонатора) и последующее возвращение энергии импульса обратно к оси резонатора.

Как видно из представленных рисунков, на больших временах электрическое поле пули остается сосредоточенным вдоль оси цилиндрического резонатора, и в этом смысле можно говорить о квазистабильном распространении световой пули. 

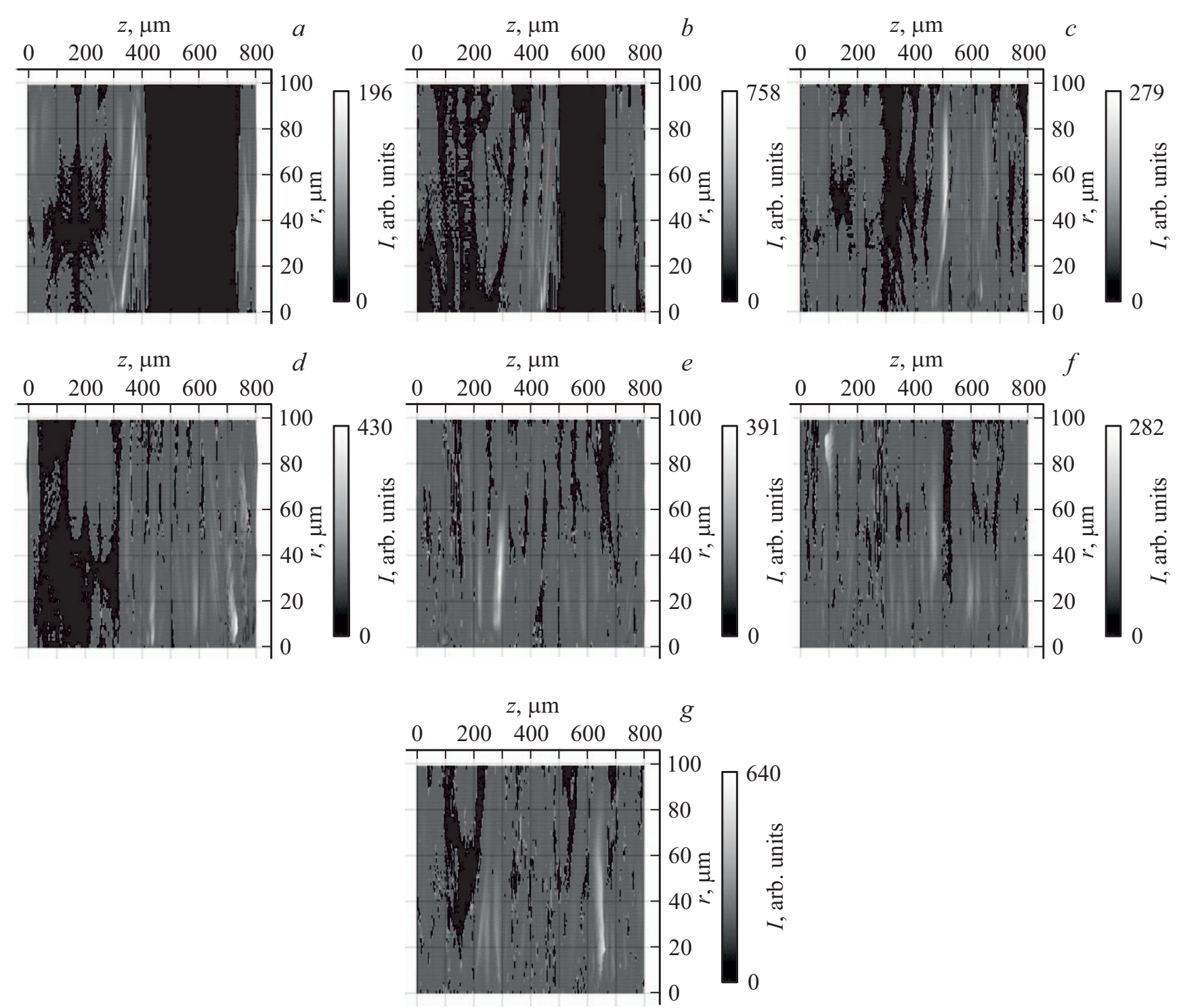

Рис. 5. Компоненты вектора Пойнтинга вдоль оси $r$ в среде упорядоченных УНТ в оптическом резонаторе в различные моменты времени $t=30(a), 40(b), 60(c), 80(d), 100(e), 120(j), 140 \mathrm{ps}(h)$.

Влияние глубины модуляции нелинейности $\alpha$ выражается в незначительном изменении формы предельно короткого импульса. Период модуляции $\chi$ оказывает существенное влияние на скорость импульса - при уменьшении периода импульс чаще отражается от узлов нелинейной среды, соответственно чаще происходит интерференция прямой и отраженных волн, и как следствие, скорость импульса падает. Очевидно, что при бесконечном периоде (отсутствии интерференции) скорость импульса будет максимальной. Данный результат был неоднократно подтвержден для импульсов фемтосекундной длительности в средах, содержащих ориентированные УНТ [22,23].

Амплитуда световой пули изменяется с течением времени, что видно на рис. 2-4. Следует заметить, что значение амплитуды меняется незначительно, в пределах нескольких относительных единиц. Отметим, что такое поведение, а именно изменение формы импульса, связано как с поперечной структурой импульса (возбуждаются внутренние колебательные моды импульса), так и с тем, что среда неоднородна (показатель преломления среды имеет пространственную модуляцию). Таким образом, бесселева структура уже не сохраняется полностью. Тем не менее локализация энергии происходит за счет многократного отражения волн от стенок резонатора и последующей интерференции.

Подводя итог, можно сказать, что в настоящей работе впервые было проведено численное моделирование динамики трехмерных предельно коротких оптических импульсов, имеющих бесселево поперечное сечение, в неоднородной среде УНТ, помещенных в оптический резонатор. В отличие от ранее известных результатов моделирование проводилось на больших временах, порядка 140 ps, это время, за которое импульс успевает пройти 
несколько дисперсионных длин (влияние дисперсии на длительность импульса: $L_{D}=T_{0}^{2} /\left|d^{2} \beta / d \omega^{2}\right|$, где $T_{0}-$ начальная длительность импульса), а вторая производная отвечает за величину дисперсии; таким образом, можно говорить о квазистабильном распространении световой пули, энергия которой остается локализованной в ограниченной пространственной области.

\section{Финансирование работы}

И.С. Двужилов, Ю.В. Двужилова, М.Б. Белоненко выражают благодарность Министерству науки и высшего образования РФ за поддержку численного моделирования в рамках государственного задания (проект № 0633-2020-0003).

\section{Конфликт интересов}

Авторы заявляют, что у них нет конфликта интересов.

\section{Список литературы}

[1] J. Durnin, J.J. Miceli, J.H. Eberly. Phys. Rev. Lett., 58, 1499 (1987). DOI: https://doi.org/10.1103/PhysRevLett.58.1499

[2] Y.S. Kivshar, G.P. Agrawal. Optical solitons: From Fibers to Photonic Crystals. (Academic press., 2003).

[3] V. Jarutis, A. Matijošius, P.D. Trapani. Opt. Lett., 34 (14), 2129 (2009). DOI: 10.1364/OL.34.002129

[4] A. Matijošius, V. Jarutis, A. Piskarskas. Opt. Express, 18 (9), 8767 (2010).

[5] J. Morris, T. Cizmar, H. Dalgarno, R. Marchington, F. Gunn-Moore, K. Dholakia. J. Opt., 12 (12), 124002 (2010). DOI: $10.1088 / 2040-8978 / 12 / 12 / 124002$

[6] J. Rosen, A. Yariv. Opt. Lett., 20 (20), 2042 (1995). DOI: $10.1364 /$ OL.20.002042

[7] I.D. Chremmos, Z. Chen, D.N. Christodoulides, N.K. Efremidis. Opt. Lett., 37 (23), 5003 (2012). DOI: $10.1364 /$ OL.37.005003

[8] J. Zhao, P. Zhang, D. Deng, J. Liu, Y. Gao, I.D. Chremmos, N.K. Efremidis, D.N. Christodoulides, Z. Chen. Opt. Lett., 38 (4), 498 (2013). DOI: $10.1364 /$ OL.38.000498

[9] S. Iijima. Nature, 354, 56 (1991). DOI: $10.1038 / 354056 a 0$

[10] S. Iijima, T. Ichihashi. Nature, 363, 603 (1993). DOI: $10.1038 / 363603 \mathrm{a} 0$

[11] M.S. Dresselhaus, G. Dresselhaus, P.C. Eklund. Science of Fullerenes and Carbon Nanotubes. (Acad. N.Y., Press, 1996).

[12] 3.Я. Косаковская, Л.А. Чернозатонский, Е.А. Федоров. Письма в ЖЭТФ., 56 (1), 26 (1992).

[13] L.A. Chernozatonsky, J.A. Barrelenes. Phys. Lett. A., 166, 55 (1992).

[14] М.К. Рокко, Р.С. Уильямс, П. Аливисатос. Нанотехнология в ближайшем десятилетии. Прогноз направления исследований. (Мир, М., 2002).

[15] Ч. Пул, Ф. Оуэнс. Нанотехнологии. (Техносфера, М., 2004).

[16] A.V. Zhukov, R. Bouffanais, M.B. Belonenko, N.N. Konobeeva, Y.V. Nevzorova, T.F. George. Eur. Phys. J. D., 69 (5), 129 (2015). DOI: 10.1140/epjd/e2015-50895-y
[17] A.V. Zhukov, R. Bouffanais, E.G. Fedorov, M.B. Belonenko. J. Appl. Phys., 114 (14), 143106 (2013). DOI: $10.1063 / 1.4824370$

[18] H. Leblond, D. Mihalache. Phys. Rev. A., 86, 043832 (2012). DOI: 10.1103/PhysRevA.86.043832

[19] М.Б. Белоненко, Н.Г. Лебедев, А.С. Попов. Письма в ЖЭТФ, 91 (9), 506 (2010).

[20] S.L. McCall, E.L. Hahn. Phys. Rev., 183(2), 457, (1969). DOI: 10.1103/PhysRev.183.457

[21] О.М. Саркисов, Э.А. Свириденков. Ж. прикл. химии, 35, 775 (1981).

[22] I.S. Dvuzhilov, Y.V. Dvuzhilova, N.N. Konobeeva, M.B. Belonenko. Rom. Rep. in Phys., 72 (3), 410 (2020).

[23] A.V. Zhukov, R. Bouffanais, M.B. Belonenko, I.S. Dvuzhilov, Y.V. Nevzorova. Appl. Phys. B., 123, 196 (2017). DOI: 10.1007/s00340-017-6767-y

[24] Y.V. Nevzorova, I.S. Dvuzhilov, S.V. Boroznin, I.V. Zaporotskova, M.B. Belonenko. EPJ Web of Conf., 161, 02018 (2017). DOI: 10.1051/epjconf/201716102008

[25] Y.V. Dvuzhilova, I.S. Dvuzhilov, A.V. Ten, E.V. Boroznina, M.B. Belonenko. Mod. Phys. Lett. B., 33 (23), 1950275 (2019). DOI: $10.1142 / \mathrm{S} 0217984919502750$

[26] R. Saito, G. Dresselhaus, M.S. Dresselhaus. Physical Properties of Carbon Nanotubes. (World Scientific, Singapore, 1998).

[27] Л.Д. Ландау, Е.М. Лифшиц. Теория поля. (Физ.-мат. лит., M., 1988).

[28] G.Ya. Slepyan, A.A. Khruttchinskii, A.M. Nemilentsau, S.A. Maksimenko. Int. J. Nanosci., 3 (3), 343 (2004). DOI: $10.1142 / \mathrm{S} 0219581 \mathrm{X} 04002152$ 